\title{
Career preferences of doctors qualifying in 1975
}

\author{
J PARKHOUSE, M K PALMER
}

British Medical fournal, 1977, 2, 25-27

Career preferences of doctors graduating in 1975 were investigated in the same way as for 1974 graduates. ${ }^{1}$ As with earlier reports ${ }^{12}$ the figures and percentages shown include a weighted allowance for tied choices-for example, medicine bracketed with another specialty as first choice of career would contribute half a first choice for medicine; when bracketed with two other specialties it would contribute one-third. The actual number of doctors expressing a particular preference, alone or tied with an alternative, cannot therefore be derived directly from the tables.

\section{Variations from the study of 1974 graduates}

NAMES AND ADDRESSES

For this study names and addresses were not obtained directly from medical schools but were derived from the GMC's fortnightly lists of provisionally registered doctors. These lists give the university of graduation (or specify the conjoint diploma) but do not distinguish individual London medical schools. Responders were asked to give their preclinical and clinical schools but 37 replies did not include this information. At the conclusion of the study figures were obtained from the University Grants Committee (UGC) for graduates from each medical school during 1975. There were slight differences from our figures, particularly in relation to London-based clinical students graduating at Oxford or Cambridge. The total UGC figure for graduates was 2752; the number of questionnaires sent out was 2734, and we received 2218 replies $(81 \%)$. The response rates for individual

University Hospital of South Manchester, Manchester M20 8LR J PARKHOUSE, MD, FFARCS, professor of anaesthetics

Department of Medical Statistics, Christie Hospital and Holt Radium Institute, Manchester M20 9BX

M K PALMER, BSC, FSS, chief medical statistician medical schools (table I) are based on UCG figures and are therefore slight underestimates.

It was clearly less satisfactory to get names and addresses in this way, for the above reasons and because only home addresses were obtained. The overall response rate was lower than previously. Nevertheless, the saving in effort to the medical schools and ourselves was considerable.

TABLE I-Response rates of graduates of various medical schools and proportion of responders known to be women

\begin{tabular}{|c|c|c|c|c|c|}
\hline $\begin{array}{l}\text { Medical } \\
\text { school }\end{array}$ & $\begin{array}{l}\text { Response } \\
\text { rate }(\%)\end{array}$ & $\begin{array}{c}\% \text { Women } \\
\text { (of res- } \\
\text { ponders of } \\
\text { known sex) }\end{array}$ & $\begin{array}{c}\text { Medical } \\
\text { school }\end{array}$ & $\begin{array}{l}\text { Response } \\
\text { rate }(\%)\end{array}$ & $\begin{array}{c}\% \text { Women } \\
\text { (of res- } \\
\text { ponders of } \\
\text { known sex) }\end{array}$ \\
\hline $\begin{array}{l}\text { Aberdeen } \\
\text { Birmingham } \\
\text { Bristol } \\
\text { Dundee } \\
\text { Edinburgh } \\
\text { Glasgow } \\
\text { Leeds } \\
\text { Liverpool } \\
\text { Manchester } \\
\text { Newcastle } \\
\text { Nottingham } \\
\text { Oxford } \\
\text { Sheffield } \\
\text { Wales }\end{array}$ & $\begin{array}{r}78 \\
79 \\
93 \\
82 \\
81 \\
73 \\
78 \\
74 \\
86 \\
83 \\
100 \\
64 \\
80 \\
79\end{array}$ & $\begin{array}{l}32 \\
35 \\
36 \\
35 \\
33 \\
29 \\
28 \\
36 \\
30 \\
32 \\
35 \\
19 \\
24 \\
34\end{array}$ & $\begin{array}{l}\text { Belfast } \\
\text { Charing Cross } \\
\text { Guy's } \\
\text { King's College } \\
\text { The London } \\
\text { The Middlesex } \\
\text { Royal Free } \\
\text { St Bartholomew's } \\
\text { St George's } \\
\text { St Mary's } \\
\text { St Thomas's } \\
\text { University College } \\
\text { Westminster }\end{array}$ & 86 & $\begin{array}{l}41 \\
20 \\
24 \\
26 \\
22 \\
25 \\
49 \\
17 \\
23 \\
24 \\
15 \\
34 \\
14\end{array}$ \\
\hline
\end{tabular}

\section{SCOPE OF STUDY}

The Queen's University of Belfast was included, and Nottingham contributed its first graduates.

\section{LOCATION OF CURRENT POST AND METHOD OF ALLOCATION}

Responders were asked where they were doing their present post (in $72 \%$ of cases the second preregistration post) in relation to their medical school. There were 709 responders holding posts outside the region of their medical school, 1132 in the city of their medical school, 351 in the NHS region of their medical school, and 26 unknown. In 
answer to the question "Did you take a post outside the region from choice?" 853 replied "yes" and 121 "no" (total 974). Presumably the excess replies (see above) referred to previously held posts outside the region. Responders were also asked their views on the best method of allocating preregistration posts.

\section{CHANCES OF SUCCESS}

The question as to chances of success was omitted, since the replies to the previous inquiry revealed confusion about the definition of "success"; we felt this to be reasonable and thought the resultant information would not justify elaborating the question.

\section{USE OF EXAMPLES}

The questionnaire to 1974 graduates stated, "You may be as specific as you like about your career preferences-for instance, if you are determined to be a paediatric neurologist or a cardiac surgeon. If it is more appropriate for you to express your preferences in broad terms, it would help us if you would use these 'mainstream' headings: medicine, surgery, obstetrics and gynaecology, general practice, psychiatry, community medicine, pathology, anaesthetics, radiology, and radiotherapy. To these you may, of course, add academic work, medical work in the armed Forces, mission work overseas, etc, etc."

The questionnaire for the present study was worded: "Be as specific or as general as you like-for instance, paediatric cardiology, mission work abroad, general practice with hospital sessions, etc."

\section{Conclusions}

There was a slightly greater proportion of women among the responders $(29.4 \%$ ) than in 1974 (table II). The number of responders born in Northern Ireland was considerably greater, but graduates of the Queen's University of Belfast were included for the first time. A considerably greater number of responders $(14.6 \%)$ did not disclose their country of birth (table III). There were less married than single responders (table IV).

Of the responders, $22.5 \%$ indicated that they had made a definite choice of career and $47 \cdot 8 \%$ said "probably."

There was a very large increase in the number of responders giving general practice combined with some other woik as a first choice of career (268 responders compared to 29 in 1974 (tables V and VI). The most popular combinations were general practice "with hospital sessions" (192), "with obstetrics" (17), "with anaesthetics" (15), and "with paediatrics" (10). The remaining combinations covered a vast range of suggestions, but the combination of general practice with community medicine accounted for only one specific preference. Community medicine

TABLE II-Percentage of women giving various specialties as first choices of career

\begin{tabular}{|c|c|c|c|}
\hline First choice of career & $\begin{array}{l}\text { Women } \\
\text { (of responders } \\
\text { of known sex) }\end{array}$ & First choice of career & $\begin{array}{c}\text { Women } \\
\text { (of responders } \\
\text { of known sex) }\end{array}$ \\
\hline \multirow{3}{*}{$\begin{array}{l}\text { Medicine } \\
\text { Paediatrics } \\
\text { Surgery } \\
\text { Obstetrics and } \\
\text { gynaecology } \\
\text { General practice } \\
\text { General practice with } \\
\text { other specialty } \\
\text { Psychiatry }\end{array}$} & $\begin{array}{l}25 \\
55 \\
10\end{array}$ & $\begin{array}{l}\text { Community medicine } \\
\text { Pathology } \\
\text { Anaesthetics }\end{array}$ & $\begin{array}{l}61 \\
34 \\
37\end{array}$ \\
\hline & $\begin{array}{l}31 \\
32\end{array}$ & $\begin{array}{l}\text { Radiology } \\
\text { Other (medical) }\end{array}$ & $\begin{array}{l}37 \\
29\end{array}$ \\
\hline & $\begin{array}{l}36 \\
32\end{array}$ & $\begin{array}{l}\text { Other (non-medical) } \\
\text { Not indicated }\end{array}$ & $\begin{array}{l}37 \\
33\end{array}$ \\
\hline
\end{tabular}

TABLE IV-Age and marital state of responders

\begin{tabular}{|c|c|c|c|c|}
\hline$\underset{\text { (years) }}{\text { Age }}$ & Married & Single & Unknown & Total \\
\hline $\begin{array}{c}\text { Unknown } \\
21 \\
22 \\
23 \\
24 \\
25 \\
26 \\
27 \\
28 \\
29 \\
30 \\
\text { Over } 30\end{array}$ & $\begin{array}{r}8 \\
3 \\
85 \\
264 \\
239 \\
91 \\
32 \\
28 \\
19 \\
13 \\
39\end{array}$ & $\begin{array}{r}12 \\
1 \\
10 \\
192 \\
474 \\
371 \\
102 \\
37 \\
21 \\
13 \\
6 \\
10\end{array}$ & $\begin{array}{r}8 \\
\\
\\
16 \\
46 \\
37 \\
20 \\
5 \\
7 \\
4 \\
5\end{array}$ & $\begin{array}{r}28(1.3 \%) \\
1 \\
13 \cdot(0.6 \%) \\
293(13.2 \%) \\
784(35.3 \%) \\
647(29 \cdot 2 \%) \\
213(9 \cdot 6 \%) \\
73(3.3 \%) \\
56(2.5 \%) \\
36(1.6 \%) \\
19(0.9 \% \%) \\
54(2.4 \%)\end{array}$ \\
\hline Total & $\begin{array}{c}821 \\
(37 \%)\end{array}$ & $\begin{array}{c}1249 \\
\left(56.30_{0}^{\circ}\right)\end{array}$ & $\begin{array}{c}148 \\
\left(6.7^{\circ}\right)\end{array}$ & $\begin{array}{c}2218 \\
(100 \%)\end{array}$ \\
\hline
\end{tabular}

TABLE $\mathrm{v}-$ Proportions of responders giving various specialties as first, second, and third choices of career

\begin{tabular}{|c|c|c|c|c|c|c|}
\hline \multirow{2}{*}{ Specialty } & \multicolumn{2}{|c|}{ First choice } & \multicolumn{2}{|c|}{ Second choice } & \multicolumn{2}{|c|}{ Third choice } \\
\hline & No & $\%$ & No & $\%$ & No & $\%$ \\
\hline $\begin{array}{l}\text { Medicine } \\
\text { Paediatrics } \\
\text { Surgery } \\
\text { Obstetrics and gynaecology } \\
\text { General practice } \\
\text { General practice with other }\end{array}$ & $\begin{array}{r}525 \\
142 \\
338 \\
76 \\
497\end{array}$ & $\begin{array}{r}23 \cdot 7 \\
6 \cdot 4 \\
15 \cdot 2 \\
3 \cdot 4 \\
22 \cdot 4\end{array}$ & $\begin{array}{r}458 \\
161 \\
214 \\
81 \\
270\end{array}$ & $\begin{array}{r}20 \cdot 6 \\
7 \cdot 3 \\
9 \cdot 6 \\
3 \cdot 7 \\
12 \cdot 2\end{array}$ & $\begin{array}{r}253 \\
72 \\
104 \\
38 \\
224\end{array}$ & $\begin{array}{r}11 \cdot 4 \\
3 \cdot 2 \\
4 \cdot 7 \\
1 \cdot 7 \\
10 \cdot 1\end{array}$ \\
\hline $\begin{array}{l}\text { specialty } \\
\text { Psychiatry } \\
\text { Community medicine } \\
\text { Pathology } \\
\text { Anaesthetics } \\
\text { Radiology/radiotherapy } \\
\text { Other (medical) } \\
\text { Other (non-medical) }\end{array}$ & $\begin{array}{r}268 \\
66 \\
14 \\
27 \\
84 \\
32 \\
113 \\
12\end{array}$ & $\begin{array}{r}12 \cdot 1 \\
3.0 \\
0 \cdot 6 \\
1 \cdot 2 \\
3 \cdot 8 \\
1 \cdot 5 \\
5 \cdot 1 \\
0 \cdot 6\end{array}$ & $\begin{array}{r}157 \\
56 \\
18 \\
29 \\
103 \\
33 \\
116 \\
15\end{array}$ & $\begin{array}{r}7 \cdot 1 \\
2 \cdot 5 \\
0 \cdot 8 \\
1 \cdot 3 \\
4 \cdot 6 \\
1.5 \\
5 \cdot 2 \\
0 \cdot 7\end{array}$ & $\begin{array}{r}84 \\
48 \\
14 \\
24 \\
56 \\
33 \\
64 \\
22\end{array}$ & $\begin{array}{l}3 \cdot 8 \\
2 \cdot 2 \\
0 \cdot 6 \\
1 \cdot 1 \\
2.5 \\
1.5 \\
2.9 \\
1 \cdot 0\end{array}$ \\
\hline Not indicated & 24 & $1 \cdot 1$ & 507 & $22 \cdot 8$ & 1182 & $53 \cdot 3$ \\
\hline
\end{tabular}

TABLE VI-Medical and surgical specialty preferences of responders

\begin{tabular}{|c|c|c|c|c|c|c|}
\hline & \multicolumn{2}{|c|}{ First choice } & \multicolumn{2}{|c|}{ Second choice } & \multicolumn{2}{|c|}{ Third choice } \\
\hline & No & $\%$ & No & $\%$ & No & $\%$ \\
\hline \multicolumn{7}{|c|}{ Medical specialties } \\
\hline General medicine & & & & & & \\
\hline (including "medicine") & 283 & $12 \cdot 8$ & 240 & $10 \cdot 8$ & 107 & $4 \cdot 8$ \\
\hline Cardiology & 16 & 0.7 & 22 & $1 \cdot 0$ & 13 & 0.6 \\
\hline Dermatology & 13 & $0 \cdot 6$ & 17 & 0.8 & 10 & $0 \cdot 5$ \\
\hline $\begin{array}{l}\text { Endocrinology } \\
\text { Geriatrics }\end{array}$ & 8 & $0 \cdot 4$ & 12 & 0.5 & & $0 \cdot 1$ \\
\hline $\begin{array}{l}\text { Geriatrics } \\
\text { Clinical haematology }\end{array}$ & $\begin{array}{r}3 \\
16\end{array}$ & $\begin{array}{l}0.1 \\
0.7\end{array}$ & 5 & $\begin{array}{l}0.2 \\
0.5\end{array}$ & 15 & $\begin{array}{l}0.5 \\
0.7\end{array}$ \\
\hline $\begin{array}{l}\text { Nephrology } \\
\text { Nelogy }\end{array}$ & $\begin{array}{r}10 \\
5\end{array}$ & 0.2 & $\begin{array}{r}11 \\
6\end{array}$ & 0.3 & 5 & $0 \cdot 2$ \\
\hline Neurology & 21 & 0.9 & 14 & 0.6 & 9 & $0 \cdot 4$ \\
\hline $\begin{array}{l}\text { Chest medicine } \\
\text { Rheumatology }\end{array}$ & 7 & $0 \cdot 3$ & 11 & 0.5 & 7 & $0 \cdot 3$ \\
\hline $\begin{array}{l}\text { Rheumatology } \\
\text { Other medical specialty }\end{array}$ & 4 & $0 \cdot 2$ & 6 & 0.3 & 5 & $0 \cdot 2$ \\
\hline $\begin{array}{l}\text { (specified) } \\
\text { Other medicl specialty }\end{array}$ & 114 & $5 \cdot 1$ & 98 & $4 \cdot 4$ & 58 & $2 \cdot 6$ \\
\hline $\begin{array}{l}\text { Other medical specialty } \\
\text { (non-specified) }\end{array}$ & 8 & 0.4 & 8 & 0.4 & 2 & $0 \cdot 1$ \\
\hline Academic medicine & 27 & $1 \cdot 2$ & 8 & 0.4 & 10 & 0.5 \\
\hline Total & 525 & $23 \cdot 7$ & 458 & $20 \cdot 6$ & 253 & $11 \cdot 4$ \\
\hline \multicolumn{7}{|c|}{ Surgical specialties } \\
\hline General surgery & & & & & & \\
\hline (including "surgery") & & $9 \cdot 2$ & 104 & 4.7 & 47 & \\
\hline Accident and emergency & 10 & 0.5 & 17 & 0.8 & 8 & $0 . \overline{4}$ \\
\hline Ear, nose, and throat & 8 & 0.4 & 9 & 0.4 & 7 & $0 \cdot \overline{3}$ \\
\hline $\begin{array}{l}\text { Neurosurgery } \\
\text { Ophthalmology }\end{array}$ & 22 & $\begin{array}{l}0 \cdot 4 \\
1 \cdot 0\end{array}$ & 12 & $\begin{array}{l}0.1 \\
0.5\end{array}$ & 3 & $0 \cdot 1$ \\
\hline Orthopaedics & 34 & 1.5 & 30 & $1 \cdot 4$ & $1 \hat{1}$ & 0.5 \\
\hline Paediatric surgery & 2 & $0 \cdot 1$ & 4 & $0 \cdot 2$ & & $0 \cdot 1$ \\
\hline $\begin{array}{l}\text { Plastic surgery } \\
\text { Th iacic/cardiac surgery }\end{array}$ & $\begin{array}{r}21 \\
4\end{array}$ & $\begin{array}{l}0.9 \\
0.2\end{array}$ & 12 & $\begin{array}{l}0.5 \\
0.2\end{array}$ & $\begin{array}{l}7 \\
2\end{array}$ & $\begin{array}{l}0.3 \\
0 \cdot 1\end{array}$ \\
\hline $\begin{array}{l}\text { Th iacic/cardiac surgery } \\
\text { Urology }\end{array}$ & 2 & 0.1 & 2 & 0.1 & & \\
\hline $\begin{array}{l}\text { Other surgical specialty } \\
\text { (spedified) }\end{array}$ & 19 & 0.9 & 14 & 0.6 & 15 & 0.7 \\
\hline Other surgical specialty & 3 & & 14 & & & \\
\hline $\begin{array}{l}\text { (non-specified) } \\
\text { Academic surgery }\end{array}$ & 3 & $0 \cdot 1$ & 4 & 0.2 & 1 & \\
\hline Total & 338 & $15 \cdot 2$ & 214 & $9 \cdot 6$ & 104 & 4.7 \\
\hline
\end{tabular}

TABLE III-Countries of birth and nationality of 2218 responders

\begin{tabular}{|c|c|c|c|c|c|c|c|c|c|c|c|}
\hline & \multicolumn{8}{|c|}{ Country of birth } & \multicolumn{3}{|c|}{ Nationality } \\
\hline & England & Wales & Scotland & $\mathrm{N}$ Ireland & $\begin{array}{c}\text { UK } \\
\text { (not specified) }\end{array}$ & Eire & $\underset{\text { (non-UK) }}{\text { Other }}$ & Unknown & British & Other & Unknown \\
\hline No $(\%)$ & $1114(50 \cdot 2)$ & $80(3.6)$ & $218(9 \cdot 8)$ & $82(3.7)$ & $147(6 \cdot 6)$ & $5(0 \cdot 2)$ & $249(11 \cdot 2)$ & $323(14 \cdot 6)$ & $2101(94 \cdot 7)$ & $104(4 \cdot 7)$ & $13(0 \cdot 6)$ \\
\hline
\end{tabular}


TABLE VII-First choices of career selected by responders from each medical school. (Figures are percentages of responders)

\begin{tabular}{|c|c|c|c|c|c|c|c|c|c|c|c|c|c|c|}
\hline & & 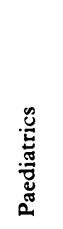 & 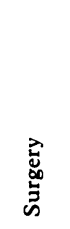 & 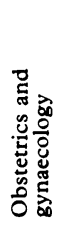 & 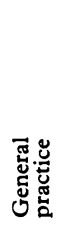 & 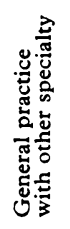 & 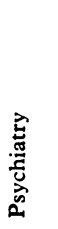 & 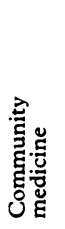 & 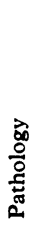 & 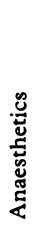 & 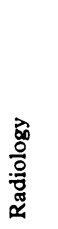 & 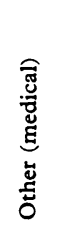 & 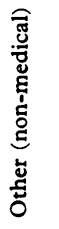 & 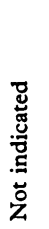 \\
\hline $\begin{array}{l}\text { Aberdeen } \\
\text { Birmingham } \\
\text { Bristol } \\
\text { Dundee } \\
\text { Edinburgh } \\
\text { Glasgow } \\
\text { Leeds } \\
\text { Liverpool } \\
\text { Manchester } \\
\text { Newcastle } \\
\text { Oxford } \\
\text { Sheffield } \\
\text { Wales } \\
\text { Charing Cross } \\
\text { Guy's Hospital } \\
\text { King's College } \\
\text { The London } \\
\text { The Middlesex } \\
\text { The Royal Free } \\
\text { St Bartholomew's } \\
\text { St George's } \\
\text { St Mary's } \\
\text { St Thomas's } \\
\text { University College } \\
\text { The Westminster } \\
\text { Belfast } \\
\text { Nottingham } \\
\end{array}$ & $\begin{array}{l}13 \cdot 8 \\
21 \cdot 3 \\
21 \cdot 0 \\
19 \cdot 2 \\
20 \cdot 7 \\
17 \cdot 1 \\
24 \cdot 6 \\
24 \cdot 4 \\
26 \cdot 8 \\
21 \cdot 4 \\
28 \cdot 8 \\
18 \cdot 1 \\
25 \cdot 5 \\
22 \cdot 9 \\
28 \cdot 8 \\
28 \cdot 6 \\
25 \cdot 6 \\
41 \cdot 9 \\
21 \cdot 7 \\
25 \cdot 1 \\
33 \cdot 3 \\
18 \cdot 5 \\
21 \cdot 3 \\
26 \cdot 4 \\
35 \cdot 7 \\
17 \cdot 0 \\
13 \cdot 5 \\
\end{array}$ & $\begin{array}{r}2 \cdot 9 \\
6 \cdot 7 \\
7 \cdot 8 \\
5 \cdot 6 \\
5 \cdot 6 \\
8 \cdot 2 \\
11 \cdot 0 \\
4 \cdot 8 \\
9 \cdot 5 \\
8 \cdot 4 \\
3 \cdot 0 \\
2 \cdot 6 \\
6 \cdot 9 \\
5 \cdot 7 \\
6 \cdot 8 \\
5 \cdot 7 \\
7 \cdot 9 \\
3 \cdot 4 \\
5 \cdot 7 \\
3 \cdot 1 \\
4 \cdot 7 \\
5 \cdot 5 \\
7.6 \\
4 \cdot 0 \\
2.0 \\
8 \cdot 1 \\
8 \cdot 1 \\
\end{array}$ & $\begin{array}{r}16 \cdot 0 \\
13 \cdot 6 \\
11 \cdot 0 \\
18 \cdot 8 \\
12 \cdot 5 \\
22 \cdot 1 \\
12 \cdot 9 \\
15 \cdot 0 \\
12 \cdot 8 \\
24 \cdot 2 \\
20 \cdot 1 \\
13 \cdot 0 \\
13 \cdot 2 \\
13 \cdot 4 \\
9 \cdot 4 \\
7 \cdot 9 \\
11 \cdot 0 \\
15 \cdot 7 \\
8 \cdot 3 \\
18 \cdot 6 \\
11 \cdot 2 \\
21 \cdot 3 \\
26 \cdot 5 \\
17 \cdot 2 \\
16 \cdot 6 \\
16 \cdot 5 \\
13 \cdot 5 \\
\end{array}$ & $\begin{array}{l}1.5 \\
2 \cdot 0 \\
1.0 \\
2 \cdot 5 \\
8 \cdot 9 \\
3 \cdot 7 \\
5 \cdot 6 \\
7 \cdot 8 \\
3 \cdot 0 \\
0.3 \\
2 \cdot 3 \\
5 \cdot 7 \\
5 \cdot 2 \\
1.4 \\
3 \cdot 4 \\
5 \cdot 7 \\
1 \cdot 4 \\
0.6 \\
1 \cdot 3 \\
4 \cdot 6 \\
4 \cdot 7 \\
3.5 \\
3 \cdot 8 \\
2 \cdot 4 \\
5.4 \\
5 \cdot 4 \\
\end{array}$ & $\begin{array}{l}28 \cdot 0 \\
25 \cdot 9 \\
25 \cdot 2 \\
22 \cdot 3 \\
28 \cdot 6 \\
20 \cdot 3 \\
18 \cdot 0 \\
21 \cdot 5 \\
21 \cdot 0 \\
22 \cdot 8 \\
16 \cdot 7 \\
24 \cdot 7 \\
23 \cdot 9 \\
30 \cdot 0 \\
22 \cdot 0 \\
25 \cdot 0 \\
21 \cdot 6 \\
12 \cdot 1 \\
36 \cdot 2 \\
15 \cdot 8 \\
12 \cdot 8 \\
20 \cdot 7 \\
11 \cdot 0 \\
12 \cdot 3 \\
18 \cdot 6 \\
33 \cdot 9 \\
37 \cdot 8 \\
\end{array}$ & 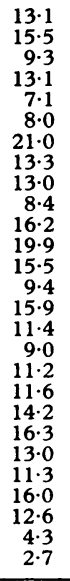 & $\begin{array}{l}5 \cdot 5 \\
3.9 \\
3 \cdot 7 \\
1 \cdot 3 \\
4 \cdot 0 \\
4 \cdot 1 \\
2 \cdot 2 \\
1.4 \\
1 \cdot 5 \\
2 \cdot 2 \\
8 \cdot 1 \\
3 \cdot 5 \\
3 \cdot 4 \\
\\
0 \cdot 6 \\
2 \cdot 9 \\
3 \cdot 2 \\
1 \cdot 1 \\
3.9 \\
4 \cdot 4 \\
4.7 \\
0 \cdot 6 \\
2 \cdot 8 \\
5.4 \\
4 \cdot 3 \\
2 \cdot 7 \\
\end{array}$ & $\begin{array}{l}1 \cdot 1 \\
0.5 \\
0.6 \\
\\
4.6 \\
1.0\end{array}$ & $\begin{array}{l}2.9 \\
0.9 \\
1.0 \\
1.0 \\
1.6 \\
1.1 \\
2.2 \\
1.7 \\
\\
2.3 \\
1.4 \\
2.1 \\
2.2 \\
2.0 \\
1.0 \\
1.7 \\
1.9 \\
2.0 \\
0.9\end{array}$ & $\begin{array}{l}5.5 \\
3.9 \\
7.0 \\
8 \cdot 8 \\
3.5 \\
6 \cdot 4 \\
1.7 \\
5.0 \\
3.0 \\
2.2 \\
1.2 \\
6.9 \\
1.7 \\
2.9 \\
2.0 \\
1.4 \\
4.2 \\
3.4 \\
1.9 \\
2.3 \\
2.3 \\
1.2 \\
3.8 \\
3.3 \\
7.0 \\
5.4\end{array}$ & $\begin{array}{l}4.4 \\
1.0 \\
0.6 \\
2.0 \\
3.3 \\
1.1 \\
2.2 \\
1.5 \\
\\
2.3 \\
1.0 \\
1 \cdot 1 \\
1.3 \\
4.9 \\
0.7 \\
1.2 \\
3.0 \\
4.0 \\
1.1\end{array}$ & $\begin{array}{r}5.1 \\
5.4 \\
10.3 \\
6.6 \\
5.1 \\
4.4 \\
0.8 \\
2.2 \\
4.8 \\
6.5 \\
1.2 \\
3.5 \\
2.3 \\
11.4 \\
2.3 \\
8.6 \\
7.2 \\
4.3 \\
5.9 \\
3.9 \\
7.0 \\
8.4 \\
10.0 \\
5.1 \\
4.4 \\
1.6 \\
5.4\end{array}$ & $\begin{array}{l}0.3 \\
1.0 \\
0.8 \\
0.7 \\
1.1 \\
0.5 \\
1.9 \\
3.2 \\
1.0\end{array}$ & $\begin{array}{l}0.9 \\
1.0 \\
1.3 \\
\\
1.1 \\
1.1 \\
0.7 \\
2.3 \\
\\
2.9 \\
2.3 \\
1.4 \\
2.1 \\
3.4 \\
1.0 \\
2.3 \\
\\
2.4 \\
4.0\end{array}$ \\
\hline Total & $23 \cdot 7$ & 6.4 & $15 \cdot 2$ & $3 \cdot 4$ & $22 \cdot 4$ & $11 \cdot 7$ & 3.0 & 0.6 & 1.2 & 3.8 & 1.5 & $5 \cdot 1$ & 0.6 & 1.0 \\
\hline
\end{tabular}

TABLE VIII-Views on allocation of preregistration posts

\begin{tabular}{l|c|c}
\hline & No & $\%$ \\
\hline Posts would best be allocated by: & & \\
(1) a national system & 196 & $8 \cdot 8$ \\
(2) own medical school & 1000 & $45 \cdot 1$ \\
(3) individual initiative & 820 & 37.0 \\
(4) combination of 2 and 3 5 combination of 1 and 2 or 1 and 3 & 160 & 7.2 \\
No reply to question & 28 & $0 \cdot 6$ \\
Posts would best be allocated both at once: & 1.3 \\
Yes & 1171 & $52 \cdot 8$ \\
No & 694 & 31.3 \\
No reply to question & 353 & 15.9 \\
\hline
\end{tabular}

as a choice of career in its own right remained unpopular (14 first choices); first choices for medicine showed an increase and anaesthetics showed a decrease.

It is difficult to escape the conclusion that the unpremeditated use of "general practice with hospital sessions" as an example in the questionnaire may have prompted a flood of responses in this direction (although the same did not apply to paediatric cardiology). If so, it is a striking example of how responders may be influenced at the preregistration stage in what they say they would like to do without, of course, necessarily being influenced to the extent of actually doing it. Here is a lesson, doubtless well known to others, in the wording of questionnaires, and a warning for all on their interpretation.

The pattern of career choices from individual medical schools (table VII) varied considerably-for example, the two schools showing the highest number of preferences for anaesthetics in 1974 yielded only one preference between them in 1975. It is important to remember such short-term variations in drawing conclusions from single samples. It is also interesting to compare these variations with the more long-term question of whether certain medical schools, over a period, tend to produce more specialists of certain kinds than others. ${ }^{3}$

There was no evidence of any increasing inclination to emigrate, the proportions of responders answering this question in accordance with the options provided being almost exactly the same as in 1974.

Only $6.5 \%$ of Belfast graduates were currently holding posts outside their own region. Within England, Scotland, and Wales, the proportion ranged from $11 \%$ of Liverpool graduates to $64 \%$ of Oxford graduates. Other medical schools with over $40 \%$ of graduates occupying posts outside the region were King's College, The Middlesex Hospital, St Bartholomew's, St George's, St Mary's, St Thomas's, and University College Hospital. Altogether, only $5.5 \%$ of responders admitted that holding a post outside the region of their medical school was not from choice.

Most responders were in favour of preregistration posts being allocated by their own medical school, or by a combination of medical school allocation and private initiative (table VIII). The suggestion of a national allocation system appealed to only $8 \cdot 8 \%$ of responders. Rather more than half of the responders $(52 \cdot 8 \%)$ felt that it would be preferable for both preregistration posts to be allocated at one time. Those in favour of private initiative alone in obtaining preregistration posts ranged from $11 \%$ to almost $70 \%$ from different medical schools, and perhaps reflected the degree of confidence in the prevailing system. Likewise, enthusiasm for allocation of both posts simultaneously ranged from around $90 \%$ in Edinburgh, Glasgow, Leeds, Liverpool, Wales, and Bristol down to between 30 and $40 \%$ of responders from other schools.

We would like to thank Miss Cynthia McLaughlin, and Mrs Mavis Howard who was responsible for much of the collation and coding of data.

\section{References}

1 Parkhouse, J, and McLaughlin, C, British Medical fournal, 1976, 2, 630.

2 Parkhouse, J, and McLaughlin, C, Lancet, 1975, 1, 1342.

${ }^{3}$ Brook, P, British fournal of Psychiatry, 1976, 128, 313.

(Accepted 11 May 1977) 